\title{
CUTANEOUS ACTIVITY OF SYSTEMIC SCLEROSIS SIMULATING PARANEOPLASTIC SYNDROME AND OPPORTUNISTIC INFECTION
}

Renata Lys Pinheiro de Mello,* , Janaina Baggio ${ }^{1}$, Carolina Cidon¹, João Calvino Soares de Oliveira ${ }^{1}$, Lucas Brandão Araújo da Silva ${ }^{1}$, Percival Degrava Sampaio-Barros ${ }^{1}$

1.Universidade de São Paulo, São Paulo (SP), Brazil.

*Corresponding author: renata.pinheiro@hc.fm.usp.br

\section{BACKGROUND}

Skin thickening, a hallmark clinical feature of systemic sclerosis (SSc), requires some differential diagnoses in the daily clinical practice of rheumatology. The authors describe a case of recurrence of important cutaneous involvement in a patient with diffuse SSc, requiring a differential diagnosis with opportunistic infection and paraneoplastic syndrome.

\section{CASE REPORT}

A female African-Brazilian patient, 50 years old, was followed at our institution since 2013, with the diagnosis of diffuse SSc. She had modified Rodnan skin score (mRSS) of 22, also presenting Raynaud's phenomenon with pitting scars, esophagopathy and mild interstitial lung disease, associated with nailfold capillaroscopy with SD pattern. The autoantibody profile revealed a coarse speckled antinuclear antibody with negative anticentromere and anti-Scl-70. Due to the severe cutaneous involvement, the patient was treated with mycophenolate mofetil for 5 years, with good clinical control. In 2018, due to the investigation of a focal change in the follow-up routine chest high resolution computed, suggestive of pulmonary tuberculosis, it was decided to discontinue mycophenolate. While the infection was ruled out, the patient evolved with a significant worsening of the skin thickening (mRSS $=38$ ), associated with generalized pruritus. Considering a cutaneous recurrence of SSc, it was indicated to start monthly pulses of cyclophosphamide. However, after the second pulse, there was appearance of disseminated skin ulcers. Due to the unfavorable evolution of the severe skin manifestation, the patient was hospitalized in January 2019 for investigation of paraneoplastic syndrome or opportunistic skin infection. The initial clinical examination during hospitalization revealed skin ulcers with honey-colored crust, with erythematous infiltrative and violaceous borders, associated with hyperchromic scleroatrophic plaques of great extension on the body surface. Laboratory investigation showed elevated inflammatory activity (acute phase reactants), with no change in the autoantibody profile or organ dysfunctions. The biopsy of the injured skin was compatible with active skin ulcer associated with sclerosing dermatitis, compatible with SSc skin activity. After extensive investigation, neoplasms and other differential diagnoses, such as vasculitis and opportunistic infections, were excluded. As the patient was discharged from the hospital with a hypothesis of severe skin recurrence, refractory to mycophenolate and cyclophosphamide, the use of rituximab in biannual doses was indicated. After the first dose of rituximab, the patient evolved with progressive improvement of the skin lesions, and rituximab was maintained for two years.

\section{CONCLUSION}

This case report shows the importance of differential diagnoses and aggressive treatment of severe skin recurrences in patients with diffuse SSc.

\section{KEYWORDS}

Systemic sclerosis, Paraneoplasia, Skin ulcer, Differential diagnosis, Infection. 